\title{
EVALUATION OF BONE REGENERATION FOR AUGMENTATION OF ALVEOLAR BONE USING 3D COMPUTER GUIDED CERAMIC SHEETS
}

\author{
Mohammed Sayed Heikal **, Mohammad abdel-hamied Shuman ${ }^{* *}$, Mohamed Abd Elsalam Khalil ${ }^{* * *}$
}

\begin{abstract}
Objective: The objective of this study was designed to evaluate bone augmentation of posterior alveolar mandibular ridge using 3D computer guided ceramic sheets as a membrane in GBR. Subjects and Methods: Seven patients were included in the present study. Preoperative clinical evaluation, and CBCT scan for ridge evaluation and planning, all patient clinically suffering from severed resorbed posterior alveolar ridge of the mandible. Measuring height and width of right and left residual alveolar ridge was performed in $\mathrm{CBCT}$ software viewer base on this digital model $3 \mathrm{D}$ zirconia sheet was designed and plan on patient CBCT then milled on CAD/ CAM 5 axis machine to the desired macroscopic shape. After surgery, clinical evaluations were done at intervals of 2 weeks, first month, $3^{\text {rd }}$ and $6^{\text {th }}$ month and directed toward the observation of the healing process, signs of inflammation, infection soft tissue dehiscence, Zirconia exposure or any complications of wound. Second surgical intervention was to remove the screws and Zirconia sheet with $\mathrm{CBCT}$ evaluation to measure alveolar ridge on both sides. Results: Results of the current showing that customized $\mathrm{Zr}$ sheet can use successfully to obtain vertical and horizontal bone augmentation well compatible with soft tissue without exposure. Conclusion: Customized Zirconia sheet act as a perfect barrier and space maintaining in GBR procedures with precise fit, and good soft tissue acceptance to Zirconia. Customized Zirconia sheet reduce chairing time and amount of graft. More predictable results can obtained by using xenograft under Zirconia sheet.
\end{abstract}

KEYWORDS: GBR, augmentation of alveolar bone, computer guided membrane.

\section{INTRODUCTION}

Adequate bone volume and quality is mandatory for the ideal placement of dental implants. When optimal anatomical conditions not exist, the situation must be changed. The most challenging bone augmentation procedures in dental implant are vertical bone augmentation. Several techniques had been developed to overcome this challenges such as autologous bone grafts, bone substitute materials, distraction osteogenesis and guided bone regeneration ${ }^{(1-5)}$.

The guided bone regeneration (GBR) has been used for bone regeneration that requires a barrier membrane to create a space and prevent invasion of soft tissue into the bone defect. This membrane must be biocompatible, flexible, and have sufficient mechanical strength. A variety of materials have been used which are non-degradable GBR membranes, such as extended polytetrafluoroethylene ePTFE, titanium-reinforced ePTFE and titanium mesh ${ }^{(6,7)}$.

However membrane exposure and infection are most frequent complications, in comparison, using degradable materials such as collagen results in superior cell adhesion and proliferation, but has poor mechanical strength and cannot maintain the space between the membrane and bone defect during surgery. Shell techniques used only a thin

\footnotetext{
* MSc Student, Oral and Maxillofacial Surgery, Faculty of Dental Medicine, Al-Azhar,Cairo, Egypt.

** Assistant professor, Oral and Maxillofacial Surgery Faculty of Dental Medicine, Assiut Al-Azhar University

*** Professor, Oral and Maxillofacial Surgery Faculty of Dental Medicine, Cairo Al-Azhar University
}

-Corresponding author: dentalheikal@gmail.com 
shell of stiff material such as autologous bone shells harvested from the angle of mandible to stabilize a particulate graft ${ }^{(2,8-13)}$.

To avoid second operation and donor site morbidity using biomaterial shells include metal-enforced membranes ,titanium membranes, allogeneic bone shells and artificial resorbable membranes have been used ${ }^{(5,14)}$.

Ceramic materials are inorganic, inert, highstrength materials and act as space-maintaining device permitting new bone formation. Individualized zirconia sheets that are made by 3-D techniques through using cone beam computed tomography (CBCT) can be used in GBR and have the ability to guide the new bone formation in any shape needed with excellent soft tissue acceptance ${ }^{(15,16)}$.
The use of zirconia sheets made by $3 \mathrm{D}$ techniques as guided bone regeneration may be of value in augmentation of posterior alveolar ridge defiance, a factor that initiate the present study.

\section{SUBJECTS AND METHODS}

Seven patients were included in the present study. They were above 48 years old. They were three males and four females. All patients were selected according to critical inclusion criteria. Preoperative clinical evaluation, and CBCT scan (Scanora 3D Soredex, Helsinki, Finland) for ridge evaluation and planning was done in all patients clinically suffering from severed resorbed posterior alveolar ridge of the mandible. Measuring height and width of right

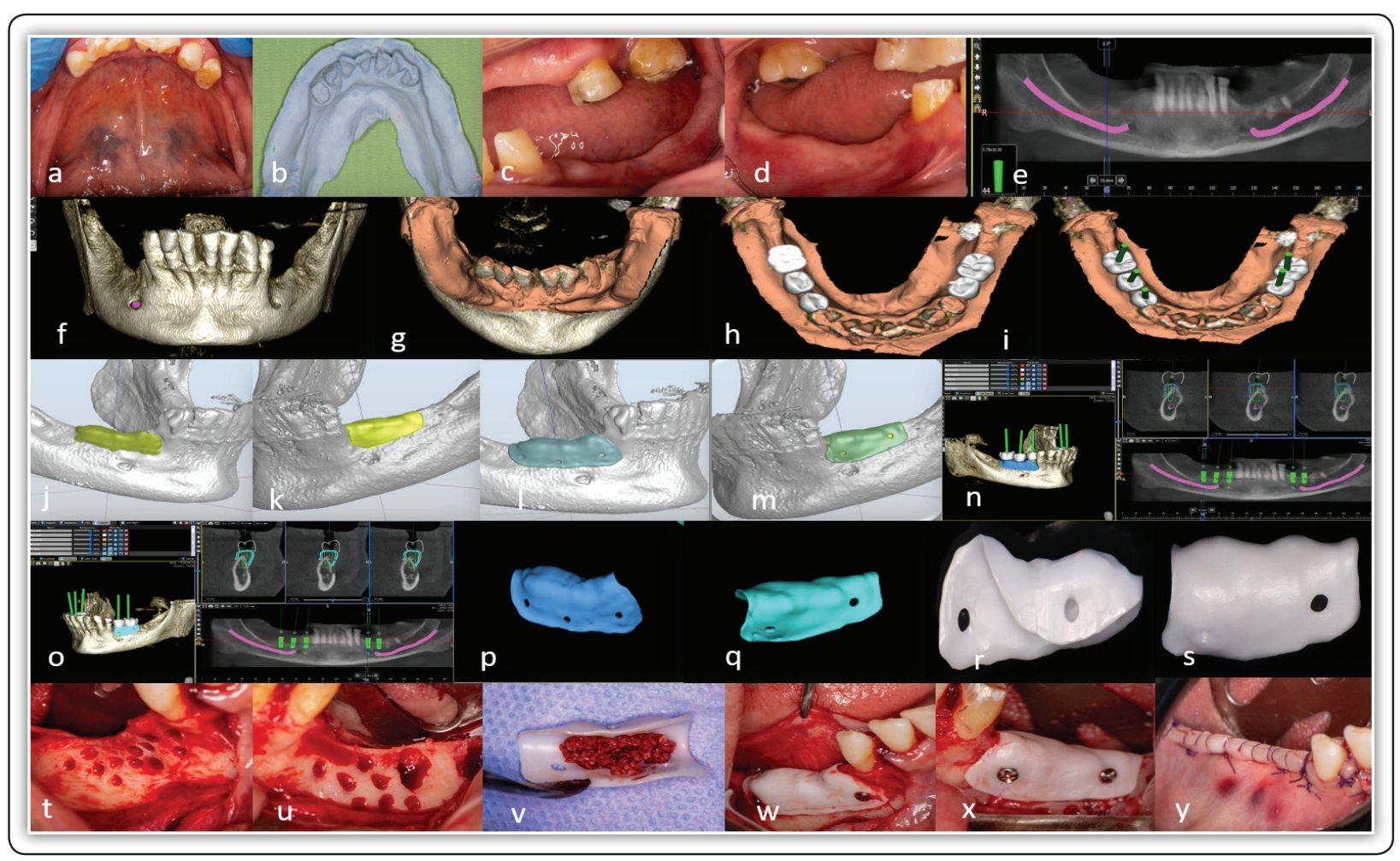

FIG (1) a; Preoperative photograph, b; mandibular cast, $\mathbf{c}$; right side, $\mathbf{d}$; left side, e; canal tracing, f; 3D generated model, $\mathbf{g}$; 3D generated model after cast registration, $\mathbf{h}$; 3D generated model with the cast and preliminary prosthetic planning, i; 3D generated model with the cast and preliminary prosthetic and implant planning, j \&k; 3D generated model with virtually augmented graft at right side left side. , I \& $\mathbf{~ m}$; membrane on 3D model with screws holes at right side left side, $\mathbf{n}$; membrane position according to prosthetic implant planning on right side, $\mathbf{o}$; of membrane position according to prosthetic implant planning on left side, $\mathbf{p}$; right membrane, $\mathbf{q}$; left membrane , $\mathbf{r}$; final $\mathrm{Zr}$ sheet after sintered and cleaning right side, s; final Zr sheet after sintered and cleaning left side , $\mathbf{t} \& \mathbf{u}$; preparation of recipient bone by decortication in Right side Left side , v; Xenografts bone chips in side ceramic sheet, w \& x; fixation of sheet by fracture screws Right side Left side $\& \mathbf{y}$; flap closure. 
and left residual alveolar ridge was performed in CBCT software viewer. Based on this digital model a 3D zirconia sheet was designed then milled on a Computer aided design/ Cumputer aided milling (CAD/CAM) 5 axis machine (Ceramill motion 2 5x, Amann Girrbach, Lichtenstein) to the desired macroscopic shape.

The procedure was conducted under local anesthesia (Articaine $\mathrm{HCl} 4 \%$, inibsa, Spain), crestal incision was made in the alveolar ridge, extended to retromolar area posteriorly and vertical releasing incisions was placed mesiobuccally at least one or two teeth anterior. Soft tissues flap were reflected subperiosteally by periosteal elevator to expose the residual ridge, recipient site preparation by bone decortication using low speed bur under irrigation. Patient specific Zirconia sheet were fixed on one side without graft and other side with xenograft using $1.5 \mathrm{~mm}$ Synthes fracture screws, then flap mobilization from both buccal and lingual side by Split thickness periosteal release incisions were completed to aid in primary tension-free closure using Vicryl 4-0.

After surgery, clinical evaluations were done at intervals of 2 weeks, first month, $3^{\text {rd }}$ and $6^{\text {th }}$ month and directed toward the observation of the healing process, signs of inflammation, infection soft tissue dehiscence, Zirconia exposure or any complications of the wound. Second surgical intervention was done to remove the screws and Zirconia sheet with CBCT evaluation to measuring alveolar ridge on both sides.

\section{RESULTS}

Seven patients suffering from bilateral alveolar ridge deficiency in mandible were included in study; these patients consisted of three males and four females with age ranged between 48.0-61.0 years with a mean age of $(55.86 \pm 4.60)$ years was treated by $3 \mathrm{D}$ ridge augmentation using customized Zirconia membrane.

In the present study, signs of inflammation, infection, evidence of pain, and evidence of soft tissue dehiscence related to operation site had not detected along the observation periods. All patients in both sides had not any Neurological disturbance throughout the study until 6 months except one case showed Neurological disturbance in Ceramic sheet side and still up to 4 months and disappear after that.

There was a statistically non-significant difference in mean vertical measurement. At 6 months, there was a statistically significant difference in mean vertical measurement. Ceramic sheet /Graft side showed a higher vertical measurement than Ceramic sheet side alone.

Preoperative there was a statistically non-significant difference in mean horizontal measurement. At 6 months there was a statistically significant difference in mean horizontal measurement. Ceramic sheet /Graft side showed a higher horizontal measurement than Ceramic sheet side alone. There was a statistically significant difference in mean Density measurement. Ceramic sheet /Graft side showed a higher Density measurement than Ceramic sheet side alone. Pre-planned there was a statistically non-significant difference in mean volumetric measurement. At 6 months there was a statistically nonsignificant difference in mean volumetric measurement. Ceramic sheet /Graft side showed a lower volumetric change measurement than Ceramic sheet side alone. 
TABLE (1): Comparison between the two groups according Vertical measurement, Horizontal measurement, Density, and Volumetric measurement.

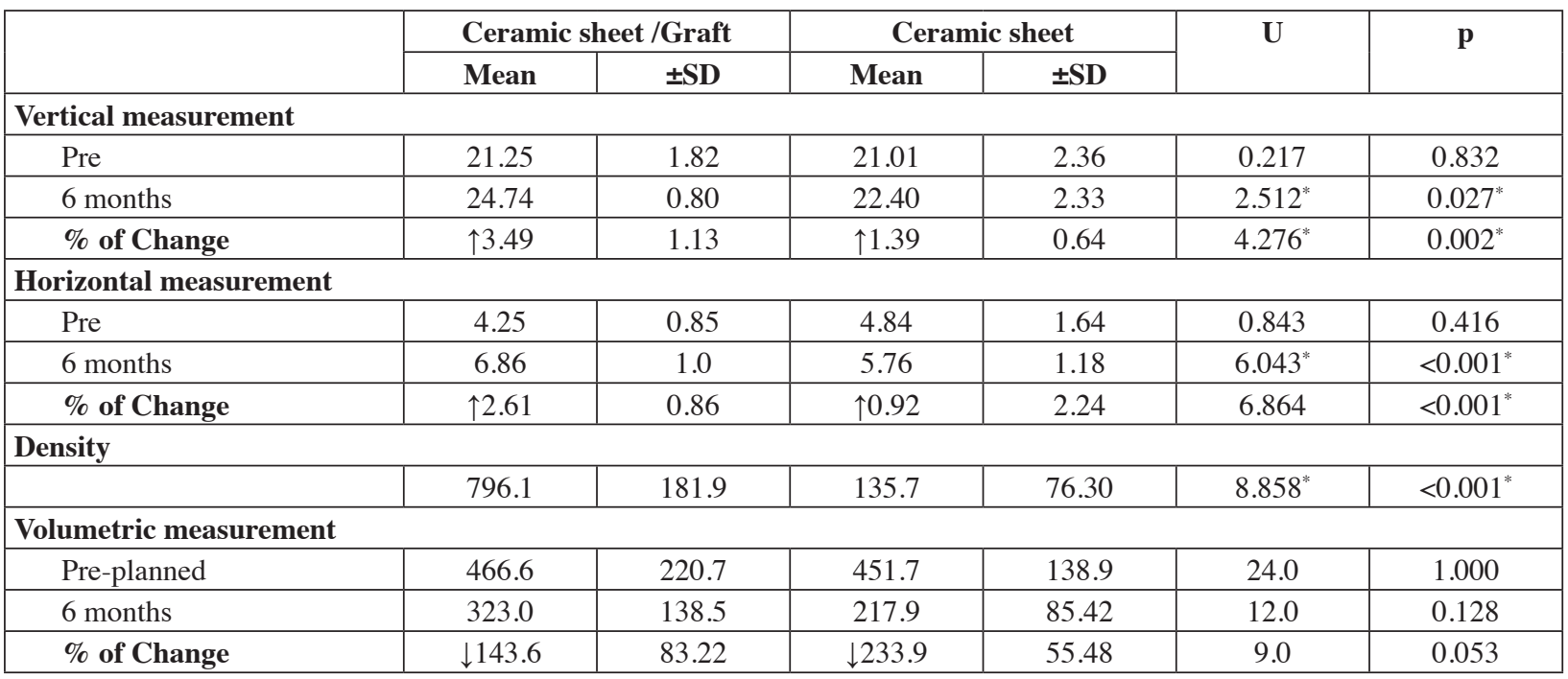

\section{$\mathbf{U}:$ Mann Whitney test $\quad$ : $p$ value for comparison between the two groups}

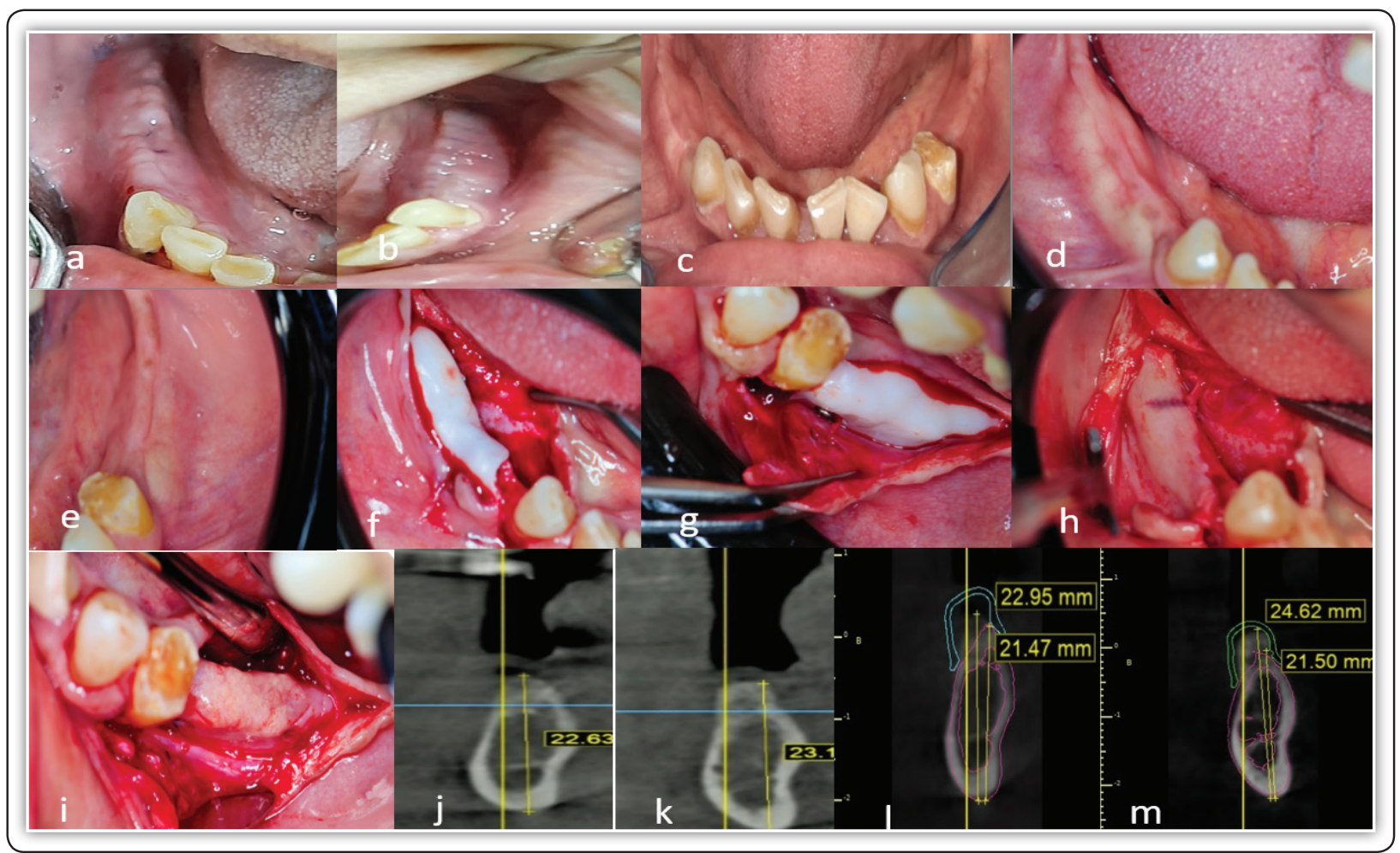

FIG (2) a; soft tissue healing two-week postoperative membrane only side, b;membrane with graft side, c; soft tissue healing 3 months postoperative, $\mathbf{d}$; soft tissue healing 6 months postoperative membrane only side, e; membrane with graft side, f; zirconia sheet after flap reflection membrane only side, $\mathbf{g}$; membrane with graft side, $\mathbf{h}$; augmented ridge after sheet removal membrane only side, i; membrane with graft side, j; CBCT 6 months post-operative in membrane only side. k; membrane with graft side, l; superimposition for preoperative CBCT cross section, 6 month post-operative and planned membrane only side, $\mathbf{m}$; membrane with graft side. 


\section{DISCUSSION}

In the present study there was a statistically significant difference in mean vertical and horizontal measurements either in membrane side or in membrane with graft side, regarding density, also there was a statistically significant difference in mean density measurement in the both sides. Based on our result, customized zirconia sheet can used in ridge augmentation procedure to reduce intraoperative time, allow for intimate and precise fit to hard tissue defects and less complicated fixation intraoperatively, and allow clinicians to precisely control the degree and location of ridge augmentation performed based on preplanned design . Zirconia appears to be a safe and effective material to serve as space maintaining device in GBR.

In the present study ceramic sheet/graft side showed no infection or dehiscence till the end of the study. One case showed neurological disturbance post-operative and continued up to four months at ceramic sheet side. We suggest that neurological disturbance was due to periosteal dissection during buccal flap mobilization.

Our results are similar to two cases studied by Craig et al. ${ }^{(17)}$, who describing the use of custom zirconia ridge augmentation matrices (CZRAM) designed with ports and filled with particulate freeze-dried bone allograft effectively to augment deficient alveolar ridges prior to dental implant placement. 3D computer-aided design (CAD) of customized zirconia rigid space maintenance devices was carried out using a baseline cone beam CT (CBCT) scan of the deficient ridges, similar to our results, no complications or wound dehiscence were encountered during healing.

Histologically evaluation by Anderud et al. ${ }^{(15)}$, showing vertical bone augmentation can be achieved using a hollow domes ceramic space maintaining devices in a rabbit caldaria model. The results suggest that the effect of the microporous structure of hydroxyapatite seems to facilitate for the bone cells to adhere to the material and that zirconia enhance a slightly larger volume of newly formed bone. The results of the current study demonstrated that ceramic space maintaining devices permits new bone formation and osteoconduction like the hollow domes.

Clinically, a soft tissue layer similar to periosteum was noted immediately underneath the Custom Zirconia and overlying the regenerated bone upon re-entry. This finding has been reported previously with the use of both custom titanium and zirconia matrices/ sheets ${ }^{(16,18,19)}$.

Guided bone regeneration (GBR) is defined as creating a space between the bones and surrounding soft tissues using a barrier that allows new bone to migrate into the space while preventing other tissue from doing so, it was originally described by Nyman et al ${ }^{(19)}$ and later by Gottlow et al ${ }^{(20)}$

Rigid space maintenance, a cornerstone of successful GBR, so using Bio absorbable membranes that lack of rigidity to maintain defect space particularly when vertical augmentation is attempted ${ }^{(21,22)}$. Rigid matrices like custom zirconia, on the other hand, guarantee the preservation of space and wound stability over the entire area to be augmented throughout the healing process. Titanium meshes can be used for creating bone for areas larger than a one-tooth gap, but usually require that particulate or block bone is transplanted to the area. Yet the use of titanium mesh has shown complications, in that it penetrates the oral mucosa, which could lead to an esthetically unsuccessful results. This seems to be avoided when placing a biodegradable membrane over the mesh to prevent the soft tissues from growing into it ${ }^{(23-26)}$.

In our study, custom zirconia was found to fit intimately to the defects, without ambiguity regarding their intended fixation position. The specificity of the fit of the sheet ensures that the 
desired gain in bone occurs in the exact site where it is needed to support the planned implant placement. Also, the precise fit allows for the minimum amount of material to be used, which lessens the size and scope of the surgery required to achieve the desired augmentation. This, in turn, can decrease the risk of morbidity to the patient and supports a minimally invasive approach to ridge augmentation.

The use of zirconia for fabrication of customized ridge augmentation matrices confers several distinct advantages over the use of titanium. Titanium matrices fabricated through additive methods such as electron beam melting or selective laser sintering require expensive machinery which limits their widespread applicability. As mentioned in a pilot study utilizing ceramic sheets, the soft tissue response to zirconia appears to be excellent. Advantage of using computer guided matrices and its clinical implications were described in a study by Sumida et al. who compared commercially available titanium mesh with customized, additively-manufactured titanium mesh ${ }^{(19)}$ The major advantage of the 3D computer guided customized ridge augmentation approach is that a pre-determined, specific amount of bone gain can be achieved that is directly congruous with the requirements of the final implant prosthetic plans.

In conclusion, Zirconia appears to be a safe and effective material to serve as a membrane used in GBR, with several advantages over titanium alloys with less complicated fixation intra-operatively, and allow clinicians to precisely control amount of ridge augmentation performed. Individualized sheet for ridge augmentation is effective methods reduce intraoperative time, allow for precise fit to hard tissue defects. The proper planning and design is key of success during use of zirconia membrane, further study needs to evaluation of mesh design membrane and different zirconia surface treatment to enhance osteoconductivite properties of zirconia.

\section{CONCLUSION}

Customized zirconiar sheet act as a perfect barrier and space maintaining in GBR procedures with precise fit. Customized zirconia sheet reduce chairing time and amount of graft. More predictable results can be obtained by using xenograft under zirconia sheet.

\section{ACKNOWLEDGEMENTS}

The authors thank Dr.Amr Ekram for his assistance in all digital and planning work .This study was partially supported by the Faculty of dentistry, Alazhar University, Egypt. The authors report no conflicts of interest related to this study.

\section{REFERENCES}

1. Sanz M, Vignoletti F. Key aspects on the use of bone substitutes for bone regeneration of edentulous ridges. Dental Materials. 2015;31(6):640-7.

2. Von Arx T, Buser D. Horizontal ridge augmentation using autogenous block grafts and the guided bone regeneration technique with collagen membranes: a clinical study with 42 patients. Clin oral implant res. 2006;17(4):359-66.

3. Roccuzzo M, Ramieri G, Bunino M, Berrone S. Autogenous bone graft alone or associated with titanium mesh for vertical alveolar ridge augmentation: a controlled clinical trial. Clin oral implant res. 2007;18(3):286-94.

4. Chiapasco M, Zaniboni M, Rimondini L. Autogenous onlay bone grafts vs. alveolar distraction osteogenesis for the correction of vertically deficient edentulous ridges: a 2-4year prospective study on humans. Clin oral implant res. 2007;18(4):432-40.

5. Urban IA, Jovanovic SA, Lozada JL. Vertical ridge augmentation using guided bone regeneration (GBR) in three clinical scenarios prior to implant placement: a retrospective study of 35 patients 12 to 72 months after loading. Int J Oral \& Maxillofac Implant. 2009;24(3):502-10.

6. Buser D, Halbritter S, Hart C, Bornstein MM, Grütter L, Chappuis V, et al. Early implant placement with simultaneous guided bone regeneration following single-tooth extraction in the esthetic zone: $12-$ month results of a prospective study with 20 consecutive patients. J Periodontol. 2009;80(1):152-62. 
7. Simion M, Dahlin C, Rocchietta I, Stavropoulos A, Sanchez R, Karring T. Vertical ridge augmentation with guided bone regeneration in association with dental implants: an experimental study in dogs. Clin Oral Implant Res. 2007;18(1):86-94.

8. Lindfors LT, Tervonen EA, Sándor GK, Ylikontiola LP. Guided bone regeneration using a titanium-reinforced ePTFE membrane and particulate autogenous bone: the effect of smoking and membrane exposure. Oral Surg, Oral Med, Oral Path, Oral Radiol, and Endodontol. 2010;109(6):825-30.

9. Degidi M, Scarano A, Piattelli A. Regeneration of the alveolar crest using titanium micromesh with autologous bone and a resorbable membrane. J Oral Implantol. 2003;29(2):86-90.

10. Watzinger F, Luksch J, Millesi W, Schopper C, Neugebauer J, Moser D, et al. Guided bone regeneration with titanium membranes: a clinical study. Br J Oral Maxillofac Surg. 2000;38(4):312-5.

11. Chiapasco M, Zaniboni M. Clinical outcomes of GBR procedures to correct peri $\square$ implant dehiscences and fenestrations: a systematic review. Clin oral implant res. 2009;20:113-23.

12. Oh TJ, Meraw SJ, Lee EJ, Giannobile WV, Wang HL. Comparative analysis of collagen membranes for the treatment of implant dehiscence defects. Clin oral implant res. 2003;14(1):80-90.

13. Khoury F, Hanser T. Mandibular bone block harvesting from the retromolar region: a 10-year prospective clinical study. Int J Oral Maxillofac Implants. 2015;30(3):688-97.

14. Nissan J, Ghelfan O, Mardinger O, Calderon S, Chaushu G. Efficacy of cancellous block allograft augmentation prior to implant placement in the posterior atrophic mandible. Clin implant dent related res. 2011;13(4):279-85.

15. Anderud J, Abrahamsson P, Jimbo R, Isaksson S, Adolfsson E, Malmström J, et al. Guided bone augmentation using ceramic space-maintaining devices: the impact of chemistry. Clin cos invest dent. 2015;7:45-53.

16. Malmström J, Anderud J, Abrahamsson P, Wälivaara D- $\AA$, Isaksson SG, Adolfsson E. Guided bone regeneration using individualized ceramic sheets. Int $\mathrm{J}$ oral maxillofac surg. 2016;45(10):1246-52.

17. Craig E. Hofferber, Cameron Beck, Peter C. Liacouras et al. Custom Zirconia Ridge Augmentation Matrix (CZRAM) for Implant Site Development: A Report of Two Cases. J Implant Advanc Clin Dent. 2018;10:6-15.

18. Sumida T, Otawa N, Kamata YU, Kamakura S, Mtsushita T, Kitagaki H, Mori S, Sasaki K, Fujibayashi S, Takemoto M. Custommade titanium devices as membranes for bone augmentation in implant treatment: Clinical application and the comparison with conventional titanium mesh. J Craniomaxillofac Surg 2015;43(10):2183-8.

19. Nyman S, Lindhe J, Karring T, Rylander H. New attachment following surgical treatment of human periodontal disease. J Clin Periodontol. 1982;9(4):290-6.

20. Gottlow J, Nyman S. Dahlin, C., A. Linde, et al.»» Healing of bone defects by guided tissue regeneration." Plastic and Reconstructive Surgery 1988; 81 (5): 672-6.

21. McAllister BS, Haghighat K. Bone augmentation techniques. J Periodontol 2007;78(3):377-96.

22. Soldatos NK, Stylianou P, Koidou VP, Angelov N, Yukna $\mathrm{R}$, Romanos GE. Limitations and options using resorbable versus nonresorbable membranes for successful guided bone regeneration. Quintessence Int 2017;48(2):131-147.

23. Lang NP, Hammerle CH, Bragger U, Lehmann B, Nyman SR. Guided tissue regeneration in jawbone defects prior to implant placement. Clin Oral Implants Res. 1994;5(2): 92-97.

24. Von Arx T, Hardt N, Wallkamm B. [The TIME technic. Local osteoplasty with micro-titanium mesh (TIME) for alveolar ridge augmentation]. Schweiz Monatsschr Zahnmed. 1995;105(5):650-663.

25. Hammerle $\mathrm{CH}$, Jung RE. Bone augmentation by means of barrier membranes. Periodontol 2000. 2003;33:36-53.

26. Abrahamsson P, Walivaara DA, Isaksson S, Andersson G. Periosteal expansion before local bone reconstruction using a new technique for measuring soft tissue profile stability: a clinical study. J Oral Maxillofac Surg. 2012;70(10):e521-e530. 